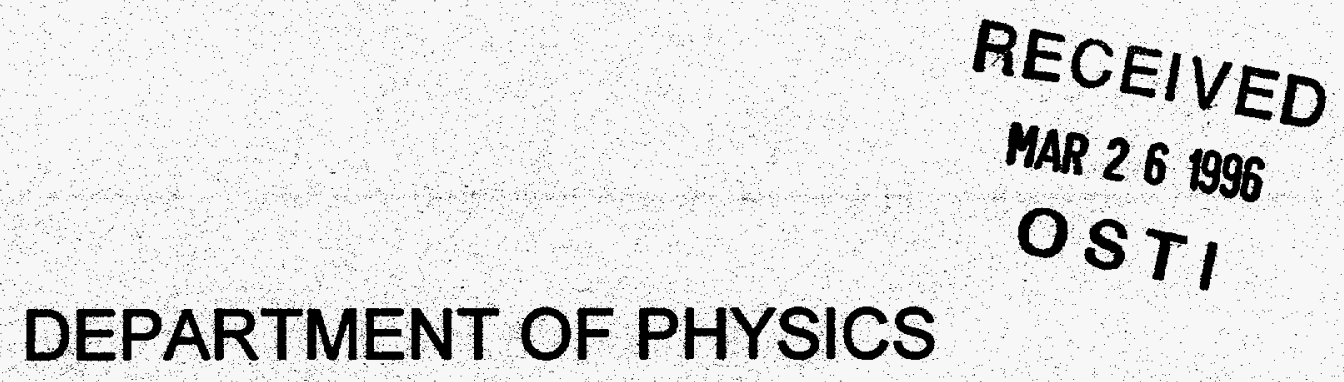

\author{
PROGRESS REPORT
}

ON

\title{
RESEARCH IN NUCLEAR PHYSICS
}

\author{
1995-1996
}

S. AYIK

COOKEVILLE, TENNESSEE 38505

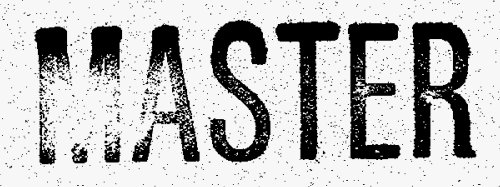

DISTAIBUTION OF THIS DOCUMENT IS UNLIMTEDPDP 


\title{
DISCLAIMER
}

This report was prepared as an account of work sponsored by an agency of the United States Government. Neither the United States Government nor any agency thereof, nor any of their employees, makes any warranty, express or implied, or assumes any legal liability or responsibility for the accuracy, completeness, or usefulness of any information, apparatus, product, or process disclosed, or represents that its use would not infringe privately owned rights. Reference herein to any specific commercial product, process, or service by trade name, trademark, manufacturer, or otherwise does not necessarily constitute or imply its endorsement, recommendation, or favoring by the United States Government or any agency thereof. The views and opinions of authors expressed herein do not necessarily state or reflect those of the United States Government or any agency thereof.

\section{STUDIES OF FLUCTUATION PROCESSES IN NUCLEAR COLLISIONS}

\author{
PROGRESS REPORT
}

MARCH 1, 1995 - MARCH 31, 1996

\author{
S. AYIK
}

DEPARTMENT OF PHYSICS

TENNESSEE TECHNOLOGICAL UNIVERSITY

COOKEVILLE, TENNESSEE 38505

MARCH 1996

PREPARED FOR THE U. S. DEPARTMENT OF ENERGY UNDER GRANT

NUMBER DE-FG05-89ER40530 


\section{TABLE OF CONTENTS}

1. Quantal Effects on Growth of Instabilities in Nuclear Matter 3

2. Collisional Damping of Giant Resonances in a Non-Markovian Approach 6

3. Medium-Modified Interaction Induced by Fluctuations 10

$\begin{array}{lr}\text { REFERENCES } & 12\end{array}$

$\begin{array}{ll}\text { APPENDIX I } & 13\end{array}$

$\begin{array}{ll}\text { APPENDIX II } & 14\end{array}$ 


\section{PREFACE}

This report summarizes the progress on grant No. DE-FG05-89ER40530 during the period March 1, 1995 to March 31, 1996.

We investigated the early evolution of the unstable nuclear matter in the spinodal zone in a quantal framework and found a significant quantum effect on the growth rates of spinodal instabilities. We have performed more accurate calculations of the collisional widths of giant resonance excitations employing a non-Markovian collision term and incorporating energy-angle dependence of the cross-section together with a diffuse nuclear surface. A transport equation was obtained by a statistical averaging of the BL equation, a statistical averaging of the BL equation, which contains a new collision term in addition to the standard form. By improving a previous treatment, we presented a derivation of this collision term. Two papers in Nucl. Phys. A, three papers in Phys. Rev. C and one paper in Phys. Lett. B have appeared. Two seminars were presented.

Most of the work done in collaboration with M. Belkacem, A. Bonasera, Ph. Chomaz, M. Colonna and Y. B. Ivanov. Their collaboration has been much appreciated.

The secretarial and accounting duties for this grant have been performed by Gloria Julian, CPS, of the TTU Department of Physics, and I wish to express my appreciation. I also wish to thank the theory groups at GSI, Darmstadt; GANIL, Caen; and LNS-INFN, Catania for their hospitality during my visits. 


\section{QUANTAL EFFECTS ON GROWTH OF INSTABILITIES IN NUCLEAR MATTER (with M. Colonna and Ph. Chomaz)}

In order to understand the early development of the spinodal instabilities, a lot of work has been carried out in the linear response framework of the semiclassical BL model [1-3]. The dynamics of the collective modes associated with density fluctuations is governed by a simple transport equation, in which the modes are agitated by a source term arising from the stochastic part of the collision integral and propagated by the mean-field. On the other hand, the work carried out earlier using the Landau kinetic model is restricted to the development of already existing fluctuations at the initial state, since this model does not contain any stochastic source term for exciting the collective modes [4]. These studies have been mostly focused on a semiclassical description of spinodal instabilities in nuclear matter. In infinite nuclear matter, the collective modes are plane waves characterized by a wave number $k$, and the characteristic frequency of a collective mode corresponding to a given wave number is determined by a semiclassical dispersion relation. It turns out that the fastest growing collective modes, which are those that will become predominant, have wave numbers that are comparable to the Fermi momentum [1]. For example, for densities $n \approx 0.3 n_{0}$ and typical temperatures $T=3-5 \mathrm{MeV}$, the wave numbers of the fastest growing modes are in the order of $k \approx 0.8 \mathrm{fm}^{-1}$, and the Fermi momentum for the same densities is $k_{\mathrm{F}} \approx 1.0 \mathrm{fm}^{-1}$. This indicates that the quantal effects associated with the mean field evolution can have an important influence on the growth of spinodal instabilities. Therefore, the quantal BL equation for the single particle density matrix $\rho(t)$ provides a more suitable framework for studying the dynamics of density fluctuations in nuclear system [5]. 
The small fluctuations $\delta \rho$ of the single particle density matrix around a finite temperature equilibrium characterized by $\rho_{0}$ are determined by the linearized quantal BL equation

$$
i \hbar \frac{\partial \delta \rho}{\partial t}-\left[h_{0}, \delta \rho\right]-\left[\delta U, \rho_{0}\right]=I_{0} \cdot \delta \rho+\delta K_{0}
$$

where $h_{0}$ is the mean field Hamiltonian at the equilibrium state, $\delta U$ denotes its fluctuating part and $I_{0} \cdot \delta \rho$ is the linearized two-body collision term. This linear response treatment provides a quantal basis for describing the early evolution of the spinodal instabilities in nuclear matter, including both the mean-field and the dissipation-fluctuation effects due to the residual interactions. In this investigation, we are mainly interested in studying the quantal effect on the growth of instabilities in finite nuclear matter, therefore we neglect r.h.s. of Eq. (1.1). Employing a plane wave representations, in which the equilibrium single particle density matrix is diagonal, $\left\langle\mathbf{p}\left|\rho_{0}\right| \mathbf{p}^{\prime}\right\rangle=\delta\left(\mathbf{p}-\mathbf{p}^{\prime}\right) \rho_{0}(\mathbf{p})$ where $\rho_{0}(\mathbf{p})$ is a finite temperature Fermi-Dirac function and carrying out a Fourier transform with respect to time, we deduce a quantal dispersion relation for the frequency of the collective mode corresponding to the wave number $k$,

$$
1=\frac{\partial U(k)}{\partial n} \int \frac{d \mathbf{p}}{(2 \pi \hbar)^{3}} \times \frac{\rho_{0}(\mathbf{p}-\mathbf{k} / 2)-(\mathbf{p}+\mathbf{k} / 2)}{\omega_{k}-\varepsilon_{\mathbf{p}+\mathbf{k} / 2}+\varepsilon_{\mathbf{p}+\mathbf{k} / 2}} .
$$

We determine the characteristic frequencies of the unstable collective modes by numerically solving the quantal dispersion relation at finite temperatures. As an example, Fig. (1.1) shows the growth rate of the unstable modes as a function of the wave number in the spinodal region, corresponding to $n=0.05 \mathrm{fm}^{-3}$ and $T=3 \mathrm{MeV}$, calculated with the effective interaction employed in Ref. [6]. The solid and the dashed lines are, respectively, the quantal and the semi-classical results with the finite range interaction. The dotted lines show the quantal results obtained with the local mean-field. The dispersion relation does not depend very strongly 
on the employed effective interaction, but is substantially modified by the quantal effect. The unstable modes are confined to a narrower range centered around a wavelength $\lambda \approx 8-10 \mathrm{fm}$, as compared to a broader range concentrated around $\lambda \approx 6 \mathrm{fm}$ in the semi-classical case. Also the maximum of the dispersion relation is reduced by the quantum effects by about a factor $2 / 3$. Therefore fluctuations take more time to develop when the quantum effects are introduced. Fig. (1.2) shows the boundary of the spinodal region in the $(n, T)$ plane corresponding to a mode with a wave length $\lambda \approx 6 \mathrm{fm}$, where solid and dashed lines are the quantal and the semi-classical results, respectively. It is seen that the spinodal region shrinks to a smaller size in the quantal case, indicating that the mode is quite suppressed by quantal effects. A paper on this topic has recently appeared in Phys. Lett. B [7], and work on a second paper is currently in progress.

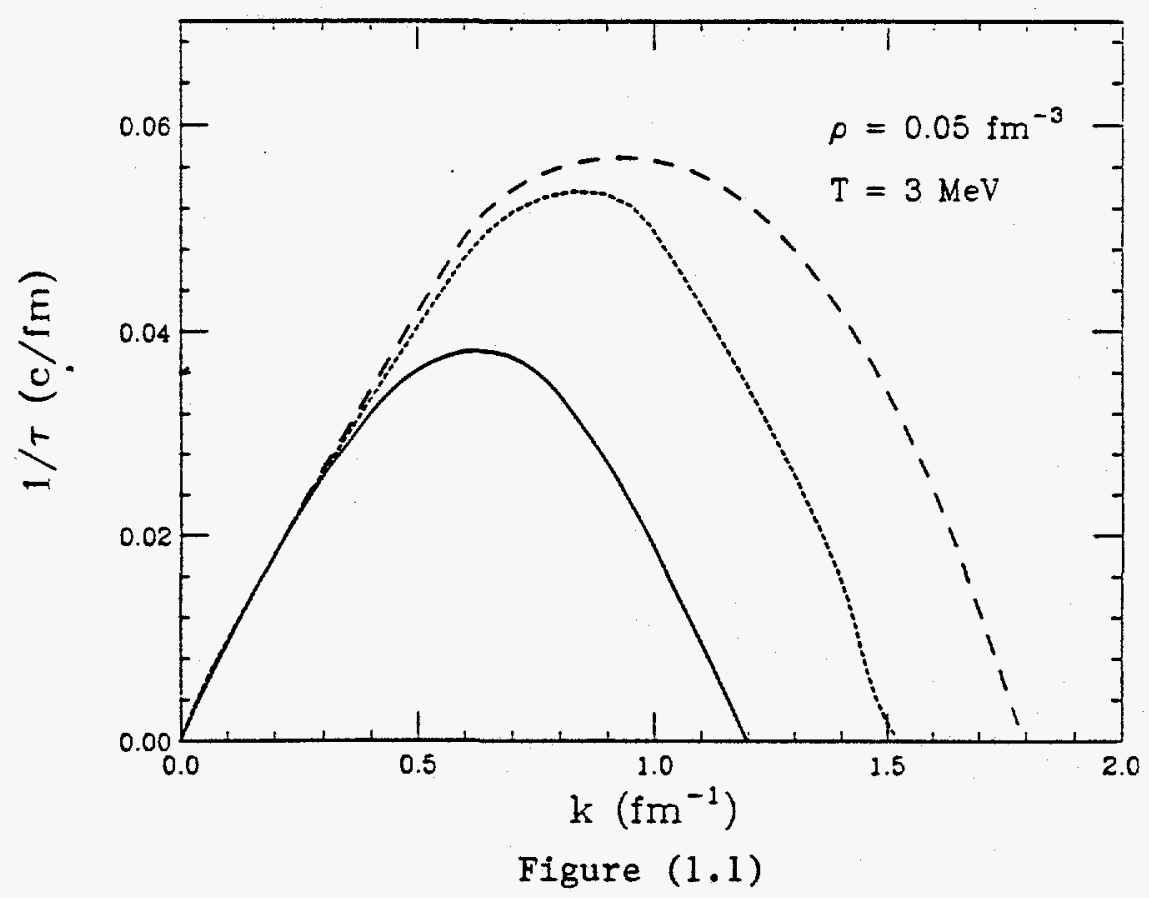




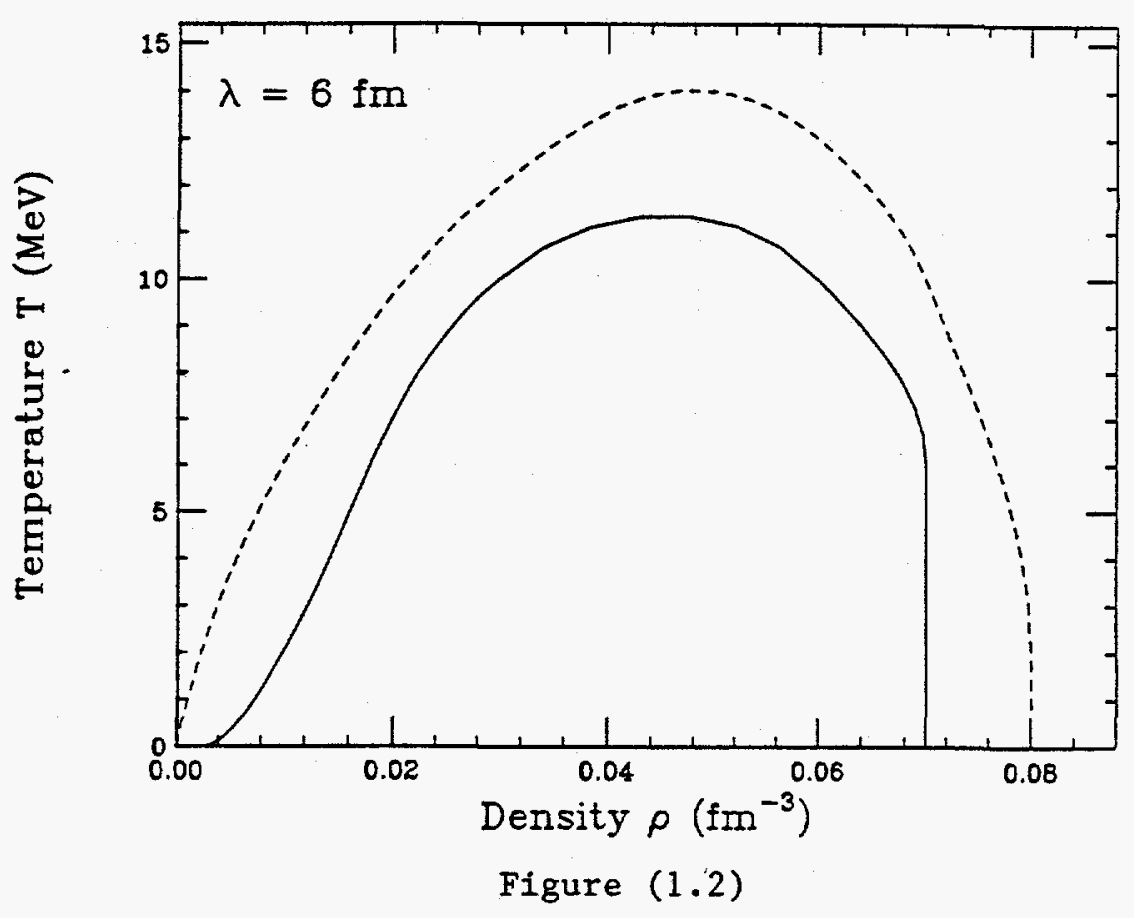

\section{COLLISIONAL DAMPING OF GIANT RESONANCES IN A NON-MARKOVIAN} APPROACH (with M. Belkacem and A. Bonasera)

Semi classical transport models of BUU-type are often employed for studying nuclear collective vibrations. Although these models give a good description of the average resonance energies, for a proper description of the collisional relaxation rate of collective vibrations, it is necessary to use these models with a non-Markovian collision term $[5,8,9,10]$. As a result, the collisional damping width of an iso-scalar collective vibration with a mean frequency $\Omega$ can be expressed as

$$
\Gamma=\frac{\int d^{3} r d^{3} p_{1} d^{3} p_{2} d^{3} p_{2} d^{3} p_{4} W Z(\Omega)(\Delta \chi)^{2} f_{1}^{0} f_{2}^{0} \tilde{f}_{3}^{0} \tilde{f}_{4}^{0}}{2 \int d^{3} r d^{3} p \chi^{2} \frac{\partial}{\partial \varepsilon} f_{0}}
$$


where $Z(\Omega)=[\delta(\Delta \varepsilon-\Omega)-\delta(\Delta \varepsilon+\Omega)] / 2 \Omega$ and $\chi \equiv(r, p, \Omega)$ denotes the distortion function of the local Fermi surface associated with the collective mode. It is possible to derive a similar expression for the collisional widths of isovector vibrations by considering the proton and neutron degrees of freedom explicitly. In order to calculate the relaxation rates, we need to know the corresponding distortion functions $\chi \equiv \chi(\mathbf{r}, \mathbf{p}, \Omega)$, which, in principle, should be determined by solving the linearized Boltzmann-Langevin equation $[8,9,10]$. However, an estimate of the collisional widths can be obtained by determining the distortion functions according to the fluid dynamical model description of collective vibrations [11]. The collective vibrations induces coherent distortions into the momentum space, and the distortion function can be expressed in terms of the velocity field $\Phi(\mathbf{r})$ as $\chi=(\mathbf{p} \cdot \nabla)(\mathbf{p} \cdot \nabla) \Phi(\mathbf{r})$. Then, it is possible to derive an analytical approximation for the collisional widths assuming a constant cross-section and neglecting the surface effects $[8,9]$.

Here, we present more accurate calculations of the collisional widths, which are carried out with energy-angle dependent cross-sections, and also by incorporating surface effects in a local density approximation. We take the velocity field associated with the giant quadrupole vibrations as $\Phi_{Q}=r^{2} P_{2}(\theta)$, and the velocity field associated with the giant monopole vibrations as $\Phi_{\mathrm{M}}=r^{2}$ and $j_{0}(k r)$, where the first and second choices correspond to the scaling description and the hydrodynamic descriptions with a wave number $k=\pi /$, respectively. We parametrize the nuclear density distribution as

$$
\rho(r)=\rho_{0}\{\exp [(r-R) / t]+1\}^{-1}
$$


where $R=1.12 A^{1 / 3}$ fm denotes the sharp nuclear radius, $\rho_{0}$ is fixed by the normalization, and $t=$ $0.545 \mathrm{fm}$ is the thickness parameter, assumed to be the same for the nuclei. The integrands in the expression (2.1) depends on $r$ through the local Fermi energy,

$$
\varepsilon_{F}(r)=\frac{\hbar^{2}}{2 m}\left[\frac{3 \pi^{3}}{2} \rho(r)\right]^{2 / 3}
$$

We employ the energy and angle dependent cross sections parametrized in Ref. [12]. and calculate the collisional widths by evaluating the momentum integrals in the expression (2.1) with the Monte-Carlo method. The result of the Monte-Carlo calculations for the quadrupole vibrations is shown as a function of mass number by solid line in Fig. (2.1). In the same figure, the analytical estimates with a constant cross-section $\sigma=40 \mathrm{mb}$ are indicated by long dashed line. In Fig. (2.2), the Monte-Carlo results for the width of monopole vibrations are shown in the hydrodynamic model by solid-line and in the scaling model by short dashed-line. In the same figure, long dashed-line indicates the corresponding analytical estimates. The Monte-Carlo calculations yield larger collisional widths than those obtained by the analytical estimate. This increase comes out as a result of the combined effect of the diffuse nuclear surface and energy-angle dependence of the cross-section. In the vicinity of the nuclear surface the Fermi motion is reduced, and hence the cross-section becomes larger, which leads to a more effective collisional damping. In the case of the monopole mode with the scaling field $r^{2}$, the Fermi surface remains spherical but oscillates radially with the monopole frequency. On the other hand the hydrodynamic velocity field $j_{0}(k r)$ induces local quadrupole distortions into the momentum space, which increases the collisional damping by about $30 \%$.

Two papers on this topic have recently appeared in Phys. Rev. C $[9,10]$. 


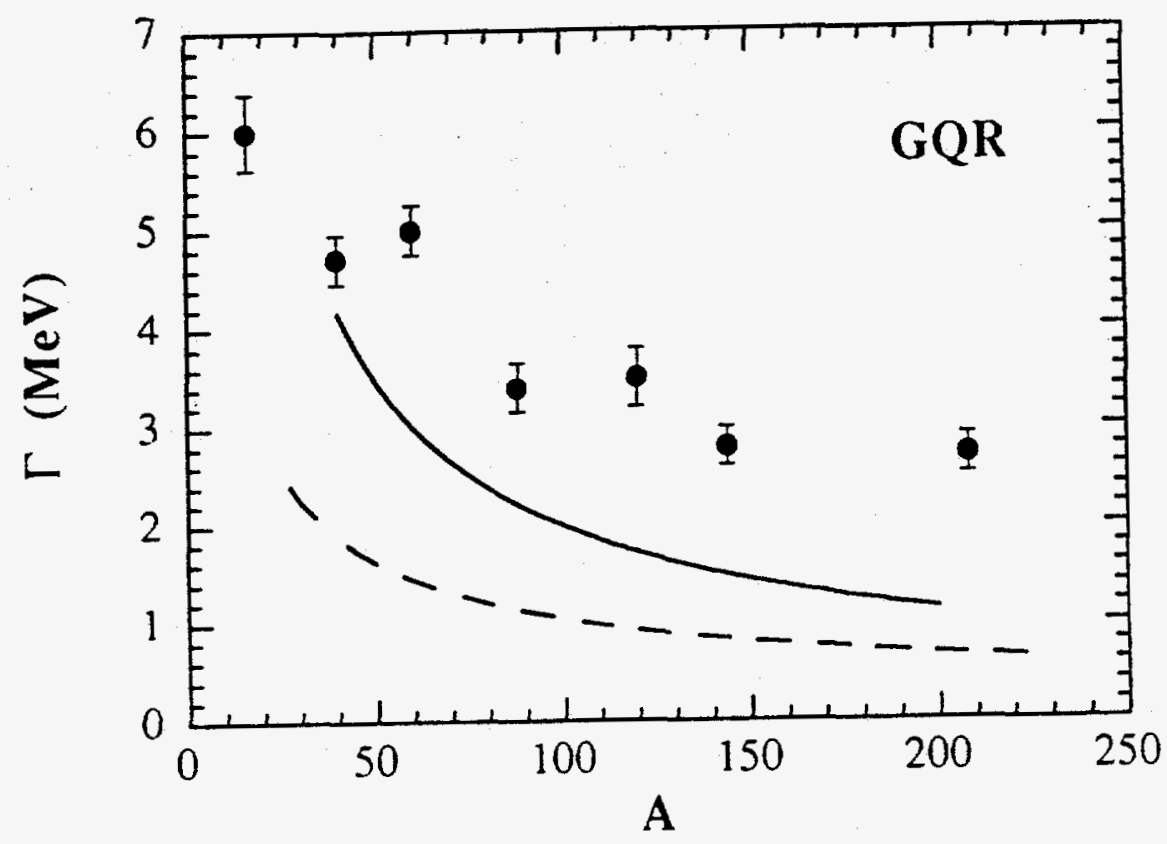

Figure (2.1)

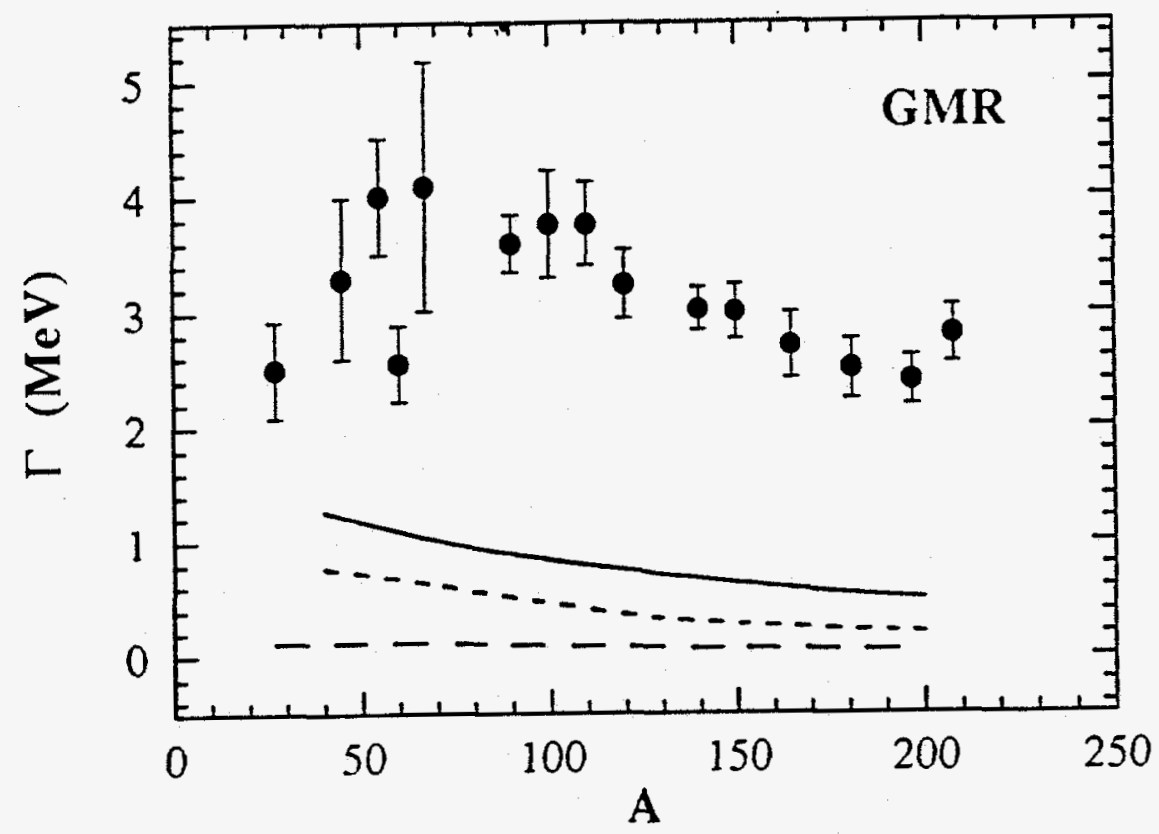

Figure (2.2) 


\section{MEDIUM MODIFIED INTERACTION INDUCED BY FLUCTUATIONS (with Y. B. Ivanov)}

In ordinary semi-classical treatment of nuclear collisions, the phase-space density $f(r, p)$ represents the average phase-space occupancy associated with an ensemble of different manybody states of the system, and the evolution is determine by the effective field calculated on the basis of the average phase-space density ignoring the mean-field fluctuations [13]. Consequently, if the system is initially prepared with a certain symmetry, the ensemble-averaged equation of motion does not permit the system to take advantage of the entropy gain associated with breaking of this symmetry. By contrast, in stochastic models of the Boltzmann-Langevin (BL) form, the fluctuations occurring in the individual densities are allowed to be propagated by their own selfconsistent Hamiltonian $[14,15]$. Then, any instabilities present may act to amplify the deviations from the average and this may lead the system to very different final configurations. An essential feature of such a dynamical process is that the ensemble average of various histories experienced by the individual systems is generally not similar to the evolution produced by the ordinary BUU dynamics. This can be seen by carrying out a statistical averaging of the BL equation,

$$
\frac{\partial}{\partial t} f(\mathbf{r}, \mathbf{p})-\{h, f\}=K_{C}(f)+K(f) .
$$

Here, $K(f)$ is the ordinary collision term of the BUU form and $K_{C}(f)$ represents an additional term, which is absent in the BUU model, arising from the correlations induced by the mean-field fluctuations,

$$
K_{C}(f)=\langle\{\delta U, \delta f\}\rangle
$$

with $\delta U$ and $\delta f$ are the deviations of the mean-field and the phase-space density from their averages, respectively. 
The collision term $K_{\mathrm{c}}$ describes an additional damping mechanism due to the coupling between the mean-field fluctuations and the single-particle motion, which may play an important role in describing transport properties of the collective modes [15]. In a previous work, a derivation of $K_{\mathrm{c}}$ was presented by assuming the amplitude of fluctuations to be small and by including only the contributions due to the collective modes [5]. In the present work, we carry out a more detailed investigation [17]. Under certain approximations $K_{\mathrm{c}}$ takes a form, similar to the Balesku-Lenard collision term in Coulomb plasmas with a transition rate,

$$
W(12 ; 34)=\frac{G(\mathbf{k}, \omega)}{(2 \pi)^{3}}\left[\frac{V(\mathbf{k})}{\varepsilon(\mathbf{k}, \omega)}\right]^{2} \delta\left(\mathbf{p}_{1}+\mathbf{p}_{2}-\mathbf{p}_{3}-\mathbf{p}_{4}\right) \delta\left(\varepsilon_{1}+\varepsilon_{2}-\varepsilon_{3}-\varepsilon_{4}\right)
$$

Here $V(\boldsymbol{k})$ represents the Fourier transform of $\partial U / \partial \mathrm{n}, \boldsymbol{\varepsilon}(\boldsymbol{k}, \omega)$ is the permittivity, and $\mathrm{G}(\boldsymbol{k}, \omega)$ is a cut-off factor with $k=p_{3}-p_{1}$ and $\omega=\left(\varepsilon_{3}-\varepsilon_{1}\right) / 2$. As demonstrated in [17], this collision term gives rise to a significant contribution to the single-particle relaxation rates in dilute nuclear systems $[18,19]$. However the treatment in its present form is valid in the domain outside of the spinodal zone.

A paper on this topic has recently appeared in Nucl. Phys. A [17]. 


\section{REFERENCES}

1. M. Colonna, Ph. Chomaz and J. Randrup, Nucl. Phys. A567 (1994) 637; M. Colonna and Ph. Chomaz, Phys. Rev. C49 (1994) 1908.

2. D. Kiderlen and H. Hofmann, Phys. Lett. B332 (1994) 8.

3. S. Ayik and J. Randrup, Phys. Rev. C50 (1994) 2947; S. Ayik, Ph. Chomaz, M. Colonna and J. Randrup, preprint LBL-35987.

4. H. Heidelberg, C. J. Pethick and D. G. Ravenhall, Phys. Rev. Lett. 61 (1988) 818.

5. S. Ayik, Z. Phys. A350 (1994) 45.

6. E. De Lima Medeiros and J. Randrup, Nucl. Phys. A529 (1991) 115.

7. S. Ayik, M. Colonna, and Ph. Chomaz, Phys. Lett. B353 (1995) 417.

8. S. Ayik and D. Boilley, Phys. Lett. B276, (1992) 263; 284, (1992) 482(E).

9. S. Ayik, M. Belkacem, and A. Bonasera, Phys. Rev. C51 (1995) 611.

10. M. Belkacem, S. Ayik and A. Bonasera, Phys. Rev. C52 (1995) 2499.

11. J. P. Blaizot, Phys. Rep. 64 (1980) 171.

12. A. Bonasera, F. Gulminelli, and J. Molitoris, Phys. Rep. 243 (1994) 1.

13. G. F. Bertsch and S. Das Gupta, Phys. Rep. 160 (1988) 190.

14. S. Ayik and C. Gregoire, Phys. Lett. B212 (1988) 269; Nucl. Phys. A513 (1990) 187.

15. J. Randrup and B. Remaud, Nucl. Phys. A514 (1990) 339.

16. G. F. Bertsch and R. A. Broglia, Oscillations in Finite Quantum Systems, Cambridge, New York (1994).

17. Yu. B. Ivanov and S. Ayik, Nucl. Phys. A593 (1995) 233.

18. G. Baym and C. J. Pethick, Landau Fermi-Liquid Theory, Wiley, New York, 1991.

19. M. T. Collins and J. J. Griffin, Nucl. Phys. A348 (1980) 63. 


\section{APPENDIX I}

$\underline{\text { PAPERS }} *$

"On Transient Effects in Violent Nuclear Collisions," E. Suraud, S. Ayik, M. Belkacem, and F. S. Zhang, Nucl. Phys. A580 (1994) 323.

"Effect of Memory Time on the Agitation of Unstable Modes in Nuclear Matter," S. Ayik and J. Randrup, Phys. Rev. C50 (1994) 2947.

'Non-Markovian Approach to Damping of Giant Monopole Resonances in Nuclei," S. Ayik, M. Belkacem and A. Bonasera, Phys. Rev. C51 (1995) 611.

"Quantal Effects on Growth of Instabilities in Nuclear Matter," S. Ayik, M. Colonna and Ph. Chomaz, Phys. Lett. $B 353$ (1995) 417.

"Medium-Modified Interaction Induced by Fluctuations," Y. B. Ivanov and S. Ayik, Nucl. A593 (1995) 233.

"Collisional Damping of Giant Resonances in a Non-Markovian Approach," M. Belkacem, S. Ayik and A. Bonasera, Phys. Rev. C52 (1995) 2499.

\section{SEMINARS}

"Effect of Finite Memory on the Transport Properties of Collective Modes," University of Catania, Catania (May 1995) and GSI, Darmstadt, Germany (June 1995).

\section{CONFERENCE CONTRIBUTIONS}

"Dynamics of Density Fluctuations in a Non-Markovian Boltzmann-Langevin Model," S. Ayik, 12th Winter Workshop on Nuclear Dynamics, Snowbird, UT (February 1996).

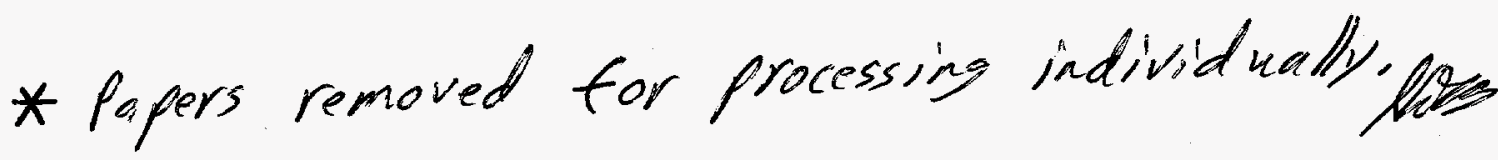


APPENDIX II

PERSONNEL

Faculty

S. Ayik, Professor

External Collaborators

M. Belkacem, Postdoc. Fellow

Laboratorio Nazionnale del Sud, INFN, Catonia, Italy

A. Bonasera, Research Staff

Laboratorio Nazionnale del Sud, INFN, Catania, Italy

Ph. Chomaz, Senior Scientist

GANIL, Caen, France

M. Colonna, Postdoc. Fellow

GANIL, Caen, France

Y. B. Ivanov, Research Staff

Kurchatov Institute, Moscow, Russia

J. Randrup, Senior Scientist

Lawrence Berkeley Laboratory, Berkeley, CA

E. Suraud, Professor

University of P. Sabatier, Toulouse, France 